Kaygl, 19(1)/2020: 152-180. Araştırma Makalesi | Research Article

Makale Geliş | Received: 20.12.2019

Makale Kabul | Accepted: 03.03.2020

Yayın Tarihi | Publication Date: 25.03.2020

DOI: $10.20981 /$ kaygi.702448

Diler Ezgi TARHAN

Dr. Öğr. Üyesi

İstanbul Gelişim Üniversitesi, Uygulamalı Bilimler Yüksekokulu, Sosyal Hizmet Bölümü, İstanbul, TR Istanbul Gelişim University, School of Applied Sciences, Social Service, İstanbul, TR

ORCID: 0000-0003-3208-9962

dilertarhan@gmail.com

\title{
Von der Analytischen Philosophie zu der Phänomenologie: Von Internamen zu den Intersinnen
}

\section{Zusammenfassung}

Das Ziel dieses Artikels ist es, was in den Philosophien von Frege und Husserl über die Beziehungen der Bedeutung der Justiz mit dem Inhalt der Justiz, den Inhalt mit der Bedeutung, die Bedeutung mit dem Objekt und das Objekt mit den Namen, gesagt wird, über die korrelative Bindung zwischen dem, was wir nennen, und dem, was wir meinen, zu diskutieren. Zu diesem Zweck wird die Husserl-Phänomenologie, mit der Bedeutung der von Frege gegründeten analytischen Tradition, beginnend mit den Dingen, die in die Sprache mit irgendeinem Name vertretet wird, seiner Meinung über Inhalt geprüft und den Unterschied der phänomenologischen Bedeutung mit der analytischen Bedeutunguntersucht. Nebenan werden auch die Nebenbedeutungen diskutiert, die mit diesem Problem in beiden Traditionen verbunden sind, und diese Diskussion, die über Husserl und Frege geführt wird, wird mit den Ansichten beider Philosophen über Konzepte wie Design, Objekt, Konzept, Denken und Phänomen erweitert.

Schlüssel Wörter: Vorstellung, Gegenstand, Begriff, Gedanke, Sachverhalt.

\section{From Analytical Philosophy to Phenomenology: From Internames to Intersense}

\begin{abstract}
The aim of this article is to discuss what is said in Frege and Husserl philosophy on the meaning of the judgment, the content of the judiciary, the meaning of the content, the meaning with the object, and the relation of the object with the names through the correlational link between what we name and what we mean. For this purpose, Husserl's phenomenology will be analyzed from the point of view of the meaning which represented with the name in the language of the analytical tradition that Frege was the founder and the difference of phenomenological meaning from analytical meaning will be investigated. On the other hand, the side meanings associated with this problem in both traditions will also be discussed and this discussion, which is carried out through Husserl and Frege, will be expanded with with the views of both philosophers about concepts such as design, object, concept, thought and fact relation.
\end{abstract}

Keywords: Design, Object, Concept, Thought, Fact Relation. 


\author{
"Etwas benennen, das ist etwas Ähnliches, \\ wie einem Ding ein Namenstäfelchen anheften."
}

(Wittgenstein 1984: 244).

\title{
Einleitung
}

In Folge des 19. Jahrhunderts bildeten sich logische Paradoxien im Bereich der Mathematik, grundlegende Veränderungen im Verständnis der Raumzeit führten bekanntlich dazu, dass feststehende philosophische Ansichten ins Schwanken gerieten. So mussten sich Frege und Husserl wie die Vertreter aller philosophischen Traditionen mit dieser Krise befassen. Wegen dieser Aufgabe entstand die Notwendigkeit, die $a$ priori Fundamente der Mathematik und die der Objektivität der Wissenschaft neu zu sichern. Dementsprechend hatten seinerzeit philosophische Traditionen bereits begonnen, die Bedeutung der Objektivität und ihre philosophischen Grundlagen infrage zu stellen. Um die Grundlagen von Wissenschaft und Philosophie objektiv bestimmen zu können, haben Philosophen verschiedene Lösungsvorschläge entwickelt. Einer dieser Vorschläge, derjenige Freges, war die mathematische Sprache zu rationalisieren, d.h. dem Bereich der Logik anzunähern, und sowohl Mathematik als auch Philosophie mithilfe einer formallogischen Sprache zu behandeln. Die Gegenstände der Mathematik und damit ihr gesamtes Gebiet wurden durch diese formale Sprache neu problematisiert, wodurch ihre Objektivität garantiert bleiben sollte. Außerdem konnte damit die a priori Geltungsbasis der Mathematik mithilfe der formalen Logik definiert werden. Der mithin wichtigste Punkt hierbei ist, dass Frege, um den Verlust der Objektivität zu vermeiden, Logik und Mathematik unabhängig von allen tatsächlichen, intuitiven und metaphysischen Elementen behandelt hat. Weil er so vorgegangen ist, stellt Freges formalisierte Sprache der Logik einen bemerkenswerten Schritt dar bzgl. der Klärung der Frage nach den objektiven Grundlagen der Wissenschaft. Doch auch wenn oder sogar deshalb weil Frege mit seiner (bloß) logischen Definition der Zahl, im Zuge seiner Arbeit "Die Grundlagen der Arithmetik"(Frege 1884) viel Anerkennung bekommen hatte, hat seine Definition der Zahl auf begrifflicher Basis in Russels 
Auseinandersetzung der Fregeschen Ausführungen ein Paradoxon in dessen Theorie sichtbar werden lassen.

Anders als Boole hat Frege auf der Suche nach der Möglichkeit von Objektivität in der Sprache qua Logik, die Logik nicht mathematisiert, sondern die Arithmetik rationalisiert (Whitesitt 1973: 27). Als andere Vertreter dieses Projekts haben Peano und Russel zu gelten, die jeweils eine rationalisierte Arithmetik vorstellen, die dem Projekt Freges ähnelt. Aus philosophischer Sicht haben sie sich der Mathematik von einer logischen Perspektive aus angenähert. Es ist bekannt, dass es neben diesem Vorschlag mithilfe einer arithmetisierten Logik die Grundlage der Mathematik zu sichern, hierfür noch weitere Vorschläge gibt wie etwa den Intuitionismus oder den Formalismus.

Wir aber konzentrieren uns auf den Begriff der Bedeutung in den Philosophien Husserls und Freges. Unsere Aufgabe wird es hier nicht sein, auf der Seite mathematischer Ansichten zur Mathematik dieser beiden Philosophen zu behandeln, sondern auf der Seite von Husserls Theorie vor Phänomenologie oder Freges Logik, sondern Husserls Ansichten zur frühen Philosophie und Freges Ansichten zur Logik. Aus diesem Grund werden wir unsere Aufmerksamkeit darauf lenken müssen, wie sie jeweils die Beziehung zwischen dem Wissenden und dem Gewussten in Bezug auf die Semantik interpretieren.

Richten wir unseren Blick auf die Folgen der Krise der Grundlagen der Mathematik, dann sehen wir, dass nicht nur neue und verschiedene Ansichten über das Thema entstanden sind, dass eine Neubefragung der Wissenschaft begonnen hat und neue Kenntnisse über die logische Sprache gesammelt wurden, und wichtiger, dass der Versuch der Wiederbelebung einer universalen, philosophischen, auf Wissenschaften gestützten Ideologie, infrage gestellt wurde. Dank der formallogischen Sprache, die von Frege in seiner "Begriffsschrift" (Frege 1964) entwickelt wurde, konnten mathematische Berechnungen mit dieser Logiksprache gemacht. Ziel dieser formalen Begriffssprache war es, eine universale mathematische Sprache zu entwickeln. Sie soll die Erforschung der wissenschaftlichen Objektivität mit sprachlichen Mitteln ermöglichen und die Objektivität der Bedeutung sichern. 
Husserl hat sich mit der phänomenologischen Untersuchung des Konzepts von Bedeutung mithilfe einer anderen Methode, d.h. auf Grundlage der Intentionalität befasst, um dieselbe objektive Basis zu erreichen. Das Hauptthema unserer Arbeit hier ist es, die Unterschiede zwischen den Philosophien Freges und Husserls hinsichtlich des Konzepts der Bedeutung zu untersuchen, und zu interpretieren wie nah sie jeweils der Objektivität gekommen sind. Zu diesem Zweck erforschen wir die Bedeutung mit Hilfe der Thema-Gegenstand Beziehung der Sprache, was unsere Arbeit mithin zu einer semantischen macht, insofern sie von einem bestimmten Urteil abhängt. Denn jede konzeptuelle Forschung, die durch sprachliche Analyse und Analyse von Urteilen durchgeführt wird, bringt uns zu einem semiotischen Punkt, sei es nun in Bezug auf die Bedeutung mathematischer Objekte oder diejenige kategorische Objekte. An diesem Punkt bedeuten die Arbeiten von Husserl und Frege zwei philosophische Traditionen und zwar die der „,arithmetischen Philosophie“ und die der „Phänomenologie“ mit einem effizienten eine Überkreuzung, der es erlaubt diese zwei Philosophien zweifellos innerhalb einer semantischen Ansicht zu vergleichen. Natürlich bedeutet diese Kreuzung nicht, dass sich die beiden Philosophen in ihren Ansichten über das Konzept der „Bedeutung“ ähnlich oder überhaupt nur nahe sind. Das Gegenteil ist der Fall und so ist es das Ziel unserer Studie, die methodologischen und inhaltlichen Unterschiede zwischen Husserls und Freges Konzept der Bedeutung offenzulegen.

Ob nun im phänomenologischen Zusammenhang von 'Erscheinung' - 'Wesen' die Rede ist oder im Zusammenhang der analytischen Philosophie von 'Sinn' - ,Bedeutung': eine Untersuchung im semantischen Raum muss Bezug auf die Intentionalitätstheorie nehmen. Bolzano wollte in der Zeit vor Frege und Husserl die Mathematik unabhängig von Anschauung begründen und suchte deshalb die Grundlage von Bedeutung in Vorstellungen als solchen. Im Zuge dessen konnte er das Feld der Semantik begründen. In seiner "Wissenschaftslehre" (Bolzano 1929) vertritt er die Ansicht, dass nur reine Logikgesetze subjektunabhängige Existenz haben. So konnte er die Möglichkeit von objektiver Bedeutung erhalten und indem er das Vertretene und die Art des Vertretens von Vorstellungen als solche trennte, hat er die Vorlagen für die Theorien von Husserl 
und Frege bereitgestellt. In Folge Bolzanos, mathematisierte Boole die Logik, woraufhin Peirce die Grundlagen der Mathematik im Feld von Semiotik untersuchen konnte.

Neben Husserl und Frege hatten Boole und Peirce sowie auch Peano, ein Zeitgenosse Freges, an Bolzano anschließend, die Bolzanos formale Sprache angeeignet, was sich konkret in der Fregeschen formalen Logik, als auch in er Husserlschen Phänomenologie zeigt.

Deshalb gelten sie alle als Schlüsselpersonen für die Entwicklung des Prozesses der Mathematisierung und Logifizierung der Philosophie, mit denen sich Frege und Husserl, wie Bolzano, Peirce und Peano in Bezug auf Freges Logik als auch in Bezug auf Husserls Phänomenologie befassen müssen. Natürlich soll diese Beziehung nicht nur als ein Einfluss, sondern auch als eine Angelegenheit für die 'Auswirkung Reaktion' gesehen werden. Frege und Husserl haben sehr verschiedene Theorien und Fachsprachen entwickelt, indem sie die intellektuellen und historischen Hintergründe der oben genannten Philosophen in Betracht gezogen haben.

\section{Die Beziehung von Sinn und Bedeutung bei Frege}

Frege war der Ansicht, dass es für die Arithmetik darauf ankommt, strenge und objektive Grundlagen für einen von allem Psychologischen und Empirischen unabhängigen Begriffsraum zu haben. Hierfür hat er die axiomatische Rechnung von Urteilen mit den entwickelten Implikationen durch die Verwendung von Quantifizierungen entwickelt. Um die Arithmetik von den Ambiguitäten natürlicher Sprachen zu befreien, hat er sie axiomatisch formalisiert. Bei der Analyse arithmetischer Urteile in der analytischen Struktur mit Hilfe von Freges rein formaler Logiksprache lässt sich nicht leugnen, dass eine semantische Analyse der arithmetischen Objekte in diesen Urteilen geboren wurde. Die semantische Analyse veranlaßte Frege, zwischen Sinn und Bedeutung $\mathrm{zu}$ unterscheiden, ebenso wie Vorstellungen ohne Wahrheitswert, die frei von allen anderen metaphysischen und empirischen Elementen aus dem Bereich der Arithmetik sind. Frege versucht, die 
zuverlässigen Grundlagen der grundlegenden Arithmetik auf der Grundlage von Objektivität zu erreichen. Er versuchte daher, die Definition für die „Anzahl“ zu finden. Er definierte einen sehr signifikanten Unterschied in der Semantik und offenbarte die Unterscheidung von Sinn und Bedeutung. „Anzahl“ definiert er als „Gedankeninhalte ohne Geist oder Empfindung.“ Freges Zahlen sind weder reale Objekte, noch mentale Vorstellungen. Frege behauptet ferner, dass Zahlen Abstraktionen sind, die aus zählbaren Mengen bestehen und definiert sie als logische Objekte, die zu einer Raumzeitlichen „Gedankenwelt“" gehören.

Nach Frege erfassen wir die Bedeutung von Zahlenobjekten im Kontext arithmetischer Urteile auf analytische Weise. Frege hat zwar dieses Einfangen vermieden, indem er die Gefahr, in den Psychologismus zu fallen, geklärt hat, aber es ist möglich, die Menge der Zahlen zu erreichen, nicht die Objekte selbst, sondern die umfassende Menge. Die Aufgabe der Philosophie besteht demnach nach Frege darin, die Funktion der Eigennamen im Kontext der Urteile zu klären, welche für die Zahlenobjekte in Zahlenangaben ausgedrückt werden. Genauer gesagt, geben sich uns Zahlenobjekte nicht direkt, sondern werden durch ihre Bedeutung erreicht, indem wir die Funktion des numerischen Kontextes der Zahlwörter an ihrer statt analysieren. Die Analyse der objektiven Beziehung zwischen 'Eigenamen' und 'Zahlobjekten', für die die Eigennamen stehen, erfordert also einerseits eine linguistische Analyse des Urteils und andererseits die Untersuchung der semantischen Grundlage von Urteilen als solchen. Dieser Forderung gemäß versucht Frege die Definition von Zahlen, ohne Rückgriff auf anschauliche, sensorische, intuitive oder fiktive Bestandteile, allein mithilfe der Logik aufzustellen.

Im Zuge der Bestimmung der Zahl als solcher wurde eine logische Analyse der Funktionen der numerischen Objekte im Satzkontext der Eigennamen durchgeführt, die die Gegenstücke der Zahlen sind. Mit dieser Unterscheidung zwischen Sinn und Bedeutung, fand Frege eine Möglichkeit die Husserlsche Phänomenologie zu fördern. Wenn wir auf diesen Punkt ein wenig näher eingehen, so stellen wir mit Frege fest, dass mit der Unterscheidung, die er zwischen Sinn und Bedeutung gefällt hat, die 
Zeichnungen immer auf Dasselbe zeigen, das sich uns auf viele verschiedene Weisen geben kann. Sicher wird jede These, die verschiedene Gegebenheitsweisen impliziert, in gewissem Sinne phänomenologisch sein müssen. Nichtsdestoweniger betrachtet die Phänomenologie die Beziehung zwischen dem Wissen und Gewusstes, dem gegeben Gegenstand in Hinsicht seiner Selbstgegebenheit und Selbstinsistenz und berücksichtigt, was und wie die Bedeutung gegeben ist. Obwohl die Unterscheidung zwischen Sinn und Bedeutung als Ergebnis der Fregeschen Bemühungen, den Begriff/ die Definition der Zahl zu klären, nicht direkt als phänomenologische Sichtweise vertreten werden kann, lässt sich sagen, dass sie dem phänomenologischen Unterschied von Erscheinung und Wesen einen Impuls gibt. Wenn wir nun zu Freges Unterscheidung von Sinn und Bedeutung zurückkehren, ist es wichtig $\mathrm{zu}$ betonen, dass Frege mit dieser Unterscheidung 'Vorstellungen' aus dem Arithmetikbereich lässt und sich von den Zahlen ausgeschlossen hat, um nicht in die Falle des Psychologismus (wie Locke und Hume) zu tappen.

Locke und Hume haben, wie wir wissen, die Psychologie der Logik und Mathematik aufgrund dieser, auf Vorstellungen basierenden, Erklärung, die durch Gedanken und durch auf Empfindungen basierende Mathematik erklärt werden, falsch verstanden. Darüber hinaus haben Hume und Locke, die eine Verbindung zwischen „Darstellungen“ und „Entwürfen“ in Richtung ,Assoziation als Verbindendes“ hergestellt haben, die naturwissenschaftliche Kausalität auf psychologische Assoziationsgesetze reduziert, weshalb sie auf die Objektivität psychologischer und empirischer Erfahrung angewiesen sind. Frege, Locke und Hume verteidigten die Idee der Zahl als 'Abstraktion', wie auch die Psychologen es getan haben. Laut Frege ist die Zahl keine fiktive Vorstellung, die von der sinnlichen Erfahrung zählbarer Objekte abstrahiert wird. Obwohl Denker wie Locke und Hume argumentieren, dass das Abstrahieren kein fiktionales Element sei, sondern viel mehr eine apriorische Idee, hat Frege es vermieden, die Zahlen vom Verstand abhängig zu machen. Aus diesem Grund versuchte Frege, der nicht nur die psychologische und empirische Grundlage, sondern auch die intuitive oder anschauliche Erklärung vermied, eine logische Erklärung der 
Zahl ohne die Objektivität preisgeben zu müssen. Frege akzeptiert die anschauliche Geometrie, jedoch keine arithmetischen Objekte. Wenn wir laut Frege versuchen, Zahlen und andere arithmetische Objekte (Mengen, Einheiten usw.) basierend auf Anschauung zu erklären, brechen wir sie von ihren Objekten auseinander. Alle die arithmetischen Objekte, die erklärt werden müssen, werden jedoch in Bezug auf ihre eigenen Objekte begriffen. Daher kritisiert Frege Psychologisten, wie Hume und Locke, dadurch dass die Fiktion sich auf die Art konzentrieren, wie der Denker spricht, anstatt sich auf den Inhalt des objektiven Denkens. Aus demselben Grund kritisierte er neben den oben genannten Psychologisten auch Formalisten wie Boole und Hilbert. Frege positionierte irrtümlicherweise, um arithmetische Objekte in ihrer Objektivität zu begreifen, diese in einem “dritten Reich“, außerhalb der Raumzeit. Denn für Frege "sind Gedanken weder Dinge der Außenwelt noch Vorstellungen.“ (Frege 1918-1919: 50). So will Frege es schaffen, sich auf den logischen Inhalt von „Gedanken“ in einem Bereich $\mathrm{zu}$ beziehen, der unabhängig ist von mentaler Fiktion und materieller Realität; so gilt ihm die Gefahr des Psychologismus als beseitigt.

Frege versuchte, die apriorischen Grundlagen der Arithmetik zu erhalten, indem er die objektive Bedeutung der Eigennamen der Zahlen im Satzzusammenhang analysierte. Nach Frege ergibt sich das Zahlenobjekt indirekt durch den Eigennamen, der das Objekt repräsentiert. Dieses „Zahlenobjekt“", das indirekt zum Wissen um es gehört, ist die Bedeutung, auf die das Wort „Anzahl“ anstelle der Zahl selbst verweist. Nach Frege bedeutet die Kenntnis eines besonderen Bedeutungstyps daher, den Gegenstand mittelbar zu identifizieren, auf den sich der Eigenname bezieht. Frege bezeichnet dieses 'Zahlenobjekt' als 'Bedeutung' und den Zahlwert des Zahlenobjekts als 'Sinn'. Mit anderen Worten: Der Eigenname als Ausdruck, der die Zahl als 'Zahlenobjekt' darstellt, gibt den Sinn des Zahlenobjekts an. Abgesehen von dieser inhaltlichen Unterscheidung zwischen Bedeutung und Sinn wird auch zwischen Sinn und Zahlen unterschieden. Während das Zahlenobjekt das sprachliche Zeichen der Zahl ist, ist das Zahlenwort oder der Eigenname der Sinn der Zahl. Mit Freges Worten: 
"Die Bedeutung eines Eigennamens ist der Gegenstand selbst, den wir damit bezeichnen; die Vorstellung, welche wir dabei haben, ist ganz subjektiv; dazwischen liegt der Sinn, der zwar nicht mehr subjektiv wie die Vorstellung, aber doch auch nicht der Gegenstand selbst ist (Frege 1994b: 44)."

Zeichen, Eigenname und schließlich 'Vorstellung' sind die individuelle und fiktionale Entsprechung des Zahlenwortes, d.h. der Sinn der Zahl. Um die Objektivität der Bedeutung zu bewahren, hat Frege die individuelle Gestaltung des Sinnes der Zahl aus ihrem objektiven arithmetischen Bereich herausgehalten. Laut ihm: "Von der Bedeutung und dem Sinne des Zeichens ist die mit ihm verknüpfte Vorstellung zu unterscheiden (Frege 1994b: 43)." So zeigt Frege, im Gegensatz zu den Psychologen, den Unterschied zwischen objektivem Sinn und subjektiver Vorstellung von vornherein. Er spaltet die Vorstellungen und erwirkt so, dass sie auf objektiver und logischer Basis arithmetischer Objekte behandelt werden können. Laut Frege:

"Wir können mit den Vorstellungen gleich die Anschauungen zusammennehmen, bei denen die Sinneseindrücke und die Tätigkeiten selbst an die Stelle der Spuren treten, die sie in der Seele zurückgelassen haben. Der Unterschied ist für unseren Zweck unerheblich (Frege, 1918-1919: 40)."

\title{
Außerdem:
}

\begin{abstract}
"Wenn Frege von den Gedanken schreibt, dass sie Eigentum von vielen sein können, so unterscheidet er sie damit scharf von den Vorstellungen, die er, wie bereits erwähnt, als rein subjektive, mit Gefühlen und Emotionen getränkte innere Bilder ansieht und von denen er ausschließt, dass sie gemeinsames Eigentum von mehreren Menschen sein können (Frege 1918-1919: 46).”
\end{abstract}

Freges konzeptioneller Eingang ${ }^{1}$ in die Definition der Zahl wurde durch die Theorie der Mengen und durch die Äquivalenz- und Gleichheitsassoziationen zwischen Mengen realisiert. Es geht uns jedoch darum, wie die Bedeutung der Zahl gelöst wird, wie die Funktion des Sinnes der Zahl gelöst wird, wie die Beziehung zwischen Bedeutung und Sinn darauf beruht und wie sich die Position auf den Sinnbegriff

\footnotetext{
${ }^{1}$ Frege benutzte die 'Humes Paarungsrelation' zwischen den Kontexten der Konzepte, um die logische Definition der Zahl zu erreichen. Der Philosoph, der die numerischen Zahlen erhält, indem er die Menge der äquivalenten und gleichförmigen Mengen findet, d.h. die Menge der äquivalenten und gleichmäßigen Mengen der Zahlen, die Menge der äquivalenten und ungleichen Mengen, basierend auf der Gleichmäßigkeit und Äquivalenzbeziehung zwischen den Deckungen der zweiten Gruppe. Er behauptet, dass sie sich unter den Mengen befinden. Die zu einem Begriff nach Frege gehörende Zahlenzahl ist daher die Deckung des zu diesem Begriff äquivalenten Begriffs.
} 
beschränkt. Aber um diese Punkte leichter verständlich zu machen, werden wir Freges Ausdrücke in Bezug auf Zahlenäquivalenz und Äquivalenz kurz erwähnen: Nach Frege ist es möglich die Gleichheitsrelation zwischen verschiedenen Zahlen wie etwa '2 ${ }^{4 \prime}$ und $4^{2,} \mathrm{zu}$ erklären. Das gelingt ihm allerdings nicht durch eine Gleichheit zwischen dem Sinn dieser Zahlen, sondern durch eine Gleichwertigkeit (Äquivalenz). Frege hat also gezeigt, dass, obwohl sich die Anzahl der Wörter in Hinsicht von Sinn unterscheidet, sie dieselbe Bedeutung haben könnten. Es gibt also keinen Unterschied in der Bedeutung, auch wenn es in den Vorschlägen, die zusammen den gleichen Wahrheitswert haben, auch wenn es "zwar eine Verschiedenheit des Sinnes zu erkennen (Frege 1998: 3)" gibt. Denn es gibt in äquivalenten Ausdrücken mit demselben Wahrheitswert einen gemeinsamen konzeptuellen Inhalt. Frege sagt über diesen gemeinsamen Inhalt folgendes:

\footnotetext{
"Ich nenne nun denjenigen Theil des Inhaltes, der in beiden derselbe ist, den begrifflichen Inhalt. Da nur dieser für die Begriffsschrift von Bedeutung ist, so braucht sie keinen Unterschied zwischen Sätzen zu machen, die denselben begrifflichen Inhalt haben (Frege 1964:3)."
}

Die logischen Erklärungen zu Relationen von Aussagen wie bspw. der Äquivalenz, Gleichheit oder Einheitlichkeit von Sätzen, tragen dazu bei, die Objektivität bei der Beschreibung der Beziehungen zwischen arithmetischen Objekten zu bewahren. Beispiel: "Wenn wir nun in der Gleichheit eine Beziehung zwischen dem sehen wollten, was die Namen ' $a$ ' und ' $b$ ' bedeuten, so schiene $a=b$ von $a=a$ nicht verschieden sein zu können, falls nämlich $\mathrm{a}=\mathrm{b}$ wahr ist. Es wäre hiermit eine Beziehung eines Dinges zu sich selbst ausgedrückt, und zwar eine solche, in der jedes Ding mit sich selbst, aber kein Ding mit einem anderen steht (Frege 1994b: 40)." Hier ist eine semantische Korrektur, die im Hinblick auf all diese Relativierungen und Details durchgeführt werden sollte, im Allgemeinen nötig für die logische Erklärung der Bedeutung und die Bewahrung ihrer objektiven Grundlagen. Aus diesem Grund versuchte Frege die Grundlagen der Arithmetik auf einer konzeptuellen Ebene zu klären, indem er jede Stufe semantisierte, und zwar insofern er die arithmetischen Objekte mit Sinn und Bedeutung verknüpfte, sodass die objektiven Prämissen der 
Bedeutung während der Untersuchung der apriorischen Arithmetikbasen nicht ignoriert wurden. Frege sagt also, dass logische Gedanken, die im Gedankenreich unabhängig von uns auf ontologische Weise positioniert werden, nur auf der Satzebene ergriffen werden können. Frege vermeidet, dieses 'ergreifende' Ding mit dem Psychologismus zu erklären, sieht den Besitz der Bedeutung eines Gegenstandes nur durch die Analyse der Funktion des Satzkontextes von Sinn, der dieses Objekt auf dem Boden darstellt.

Frege bewertete nicht nur logische Objekte, sondern auch Vorschläge in Bezug auf Sinn und Bedeutung. Laut ihm ist der Wahrheitswert der Bedeutung die objektive Bedeutung, also die sprachliche Aussage hingegen ist der Sinn. Wenn sich der Sinn ändert bleibt der Wahrheitswert derselbe, aber die Bedeutung nicht. Daher kann sich in einem Urteil die logische Form, also der Sinn verändern, aber der Wahrheitswert, der dem Satz entspricht, ändert sich nicht. Solche Situationen, in denen sich die Bedeutung trotz dem Sinn nicht ändert, zeigen, dass Sinn nicht der Träger der Bedeutung ist. Bedeutung ist ontologisch vom Sinn unabhängig, wird aber durch den Sinn bekannt gemacht. Wenn Bedeutung von Sinn nicht ontologisch unabhängig wäre, wäre es nicht möglich, von bedeutungslosen Ausdrücken zu sprechen, obwohl sie einen Sinn ergeben mögen. Allerdings gibt es solche Ausdrücke und sie können sinnvoll sein, obwohl sie keine Bedeutungen haben, wie der folgende Satz bspw. deutlich macht: "Die Worte 'der von der Erde am weitesten entfernte Himmelskörper' haben einen Sinn; ob sie aber auch eine Bedeutung haben, ist sehr zweifelhaft. [...] Dadurch also, daß man einen Sinn auffasst, hat man noch nicht mit Sicherheit eine Bedeutung (Frege 1994b: 42)." So erfordert die Anwesenheit eines Sinnes nicht das Vorhandensein einer Bedeutung. Laut Frege liegt die Sache bei arithmetischen Objekten jedoch anders, da arithmetische Objekte als logische Objekte charakterisiert werden, die sowohl einen Sinn, als auch eine Bedeutung haben. Frege aber stellt fest, dass alle nicht-numerischen Objekte einen Sinn haben müssen, um bedeutungsvoll zu sein. In anderen Worten, gibt es solche Objekte, die zwar Sinn, aber keine Bedeutung haben, womit sie keinen Wahrheitswert erhalten, aber dank nur dem Sinn trotzdem sinnvoll sein können. Ebenso sagt Frege, dass Begriffe nicht notwendig an eine Bedeutung gebunden sind, obwohl sie einen Sinn 
haben; also Begriffe, die nicht thematisiert sind, d.h. leere Haufen sind und nicht einem Gegenstand unter enthalten sind.

Frege nennt Namen "welche Eigennamen ohne Bedeutung enthalten (Frege 1994b:47)" 'gefälschte Eigennamen'. Diese haben keinen Wahrheitswert, also keine Bedeutung, sind aber inhaltsvolle Ausdrücke. Der Grund hierfür ist, dass sie einen Sinn haben. 'Warum wollen wir denn aber, dass jeder Eigenname nicht nur einen Sinn, sondern auch eine Bedeutung habe? Warum genügt uns der Gedanke nicht? Weil und soweit es uns auf den Wahrheitswert ankommt (Frege 1994b: 48)”. Hier erfüllen die Zahlenangaben diesen Anspruch, d.h., dass sie alle Sinn und Bedeutung besitzen, und enthalten daher eine Erklärung über einen Begriff. Frege glaubt, dass Begriffe der 'sekundären Ebene' in den Zahlenwissenschaften eine Aussage über 'Begriffe auf der primären Ebene' machen sollten. Genauer gesagt, der allgemeine Begriff des bspw. Zählens ist ein Begriff der zweiten Ebene, der unter einen Begriff der ersten Ebene fällt.

Das oben erwähnte Konzept von 'Begriffen auf der primären Ebene' und 'Begriffe aus der sekundären Ebene' sind Konzepte, die keine leeren Mengen sind, d.h. Sie enthalten sowohl Sinn als auch Bedeutung. Was den Unterschied zwischen diesen beiden Konzepten anbelangt, sind Objekte unter dem Begriff der ersten Ebene; Begriffe fallen unter die sekundäre Ebene. Mit anderen Worten: Elemente von Begriffen der ersten Ebene sind Objekte, während Elemente von Begriffen der zweiten Ebene Begriffe sind. Wenn Begriffe der ersten Ebene unter die Begriffe der zweiten Ebene fallen, d.h., wenn sie ihre Mitglieder sind, kann der allgemeine Begriff der einzelnen Zahl in den Zahlenwissenschaften identifiziert werden. Frege erklärt in einer logischen Ebene die Bedeutungstheorien der Arithmetik mit Hilfe einer Mengentheorie, die auf der Unterscheidung zwischen Begriff und Objekt über die Beziehung des Umfassens und des Begriffsinhaltes beruht. In dieser begrifflichen Erklärung gibt es keine anschaulich, intuitiv, psychologisch oder empirisch begründete Grundlage. Diese Bemühung, die nur unter der Logik durchgeführt wurde, hat die Entwicklung einer Theorie der analytischen und semantischen Qualitäten zur Verfügung gestellt, um die zugrundeliegenden Bedeutungsgrundlagen der Objektivität $\mathrm{zu}$ bestimmen. Diese 
Theorie auch $\mathrm{zu}$ wichtigen Diskussionen über das Problem der Bedeutung in der Sprache der formalen Logik.

Um das Bedeutungsproblem bei Frege und Husserl zu vergleichen, sollte zunächst Freges Unterscheidung von Sinn und Bedeutung hinsichtlich der Unterscheidung von Begriff und Argument untersucht werden, um daraufhin die Husserlsche Unterscheidung zwischen Noesis und Noema in den Blick zu nehmen.

Laut Frege sendet eine Funktion wie f (x) einen anderen Wahrheitswert an das Argument, das in es prädiziert wird, d.h. den Wert von $\mathrm{x}$, der für die Funktion ausgefüllt wird. Das heißt die Bedeutung einer Funktion ist wahr oder falsch, je nach dem Argument, also den Begriffen die unter sie fallen. Es gibt Begriffe denen Objekte unterliegen und Begriffe denen wiederum Begriffe unterliegen. Daher ist die Tatsache, dass ein Begriff, also eine Funktion, eine Bedeutung haben kann, bzw. einen unterliegenden Wert hat, das heißt, dass sie keine leere Menge ist, sondern sie schon vom einen Argument gefüllt wurde. Aus diesem Grund ist für Frege die Konzeption des Begriffs identisch mit dem Wert einer Funktion. Jedoch ist $\mathrm{zu}$ beachten, “daß das Argument nicht mit zur Funktion gehört, sondern mit der Funktion zusammen ein vollständiges Ganzes bildet; denn die Funktion allein ist unvollständig, ergänzungsbedürftig oder ungesättigt zu nennen (Frege 1994a: 21)." Ein Beispiel: legen wir "die Hauptstadt des x" zugrunde, so erhalten wir etwa den Funktionswert "Berlin" für das Argument 'deutsches Reich (Frege 1994a: 29).” Offensichtlich hat Frege diese Formulierung so nicht nur für Urteile und deren wahrheitsgemäße Werte verwendet, sondern auch für Begriffe und deren Unterordnung unter ein Objekt. Während also ein Begriff mit Bedeutung an einen Gegenstand gesendet wird, sendet ein Urteil mit Bedeutung es zu einem Wahrheitswert. Gesetze, die festlegen welchen Wahrheitswert ein Urteil als Prädikat hat und welche Funktion es nach dem Argument haben wird, sind Wahrheitsgesetze und laut Frege werden diese von der Logik geleitet. Frege meint, dass die Wahrheit zur Bedeutung eines Urteils prädiziert wird. Daher plädiert Frege dafür, dass die Logik, 'die allgemeinsten Wahrheiten' in der logischen Struktur, Objektivität garantiert. Seine Ansicht ist parallel zum Kontext der Begriff des Kontextes bei Kant, 
wenn er sagt, dass begriffliche Inhalte im Rahmen eines Urteils ergriffen werden können. Frege schreibt von diesen konzeptionellen Inhalten, die er 'Gedanken' nennt, folgendes: "Ich verstehe unter Gedanken nicht das subjektive Tun des Denkens, sondern dessen objektiven Inhalt, der fähig ist, gemeinsames Eigentum von vielen zu sein (Frege 1994b: 46)." Frege meint, obwohl sich die Urteilsformen auf jeder Ebene ändern, dass die „Wahrhaftigkeit“ dieselbe bleibt, weil alle Menschen mit verschiedenem Sinn die gleichen Gedanken ausdrücken können. Deshalb "ersetzen wir nun in ihm ein Wort durch ein anderes von derselben Bedeutung, aber anderem Sinne, so kann dies auf die Bedeutung des Satzes keinen Einfluß haben (Frege 1994b: 47)." Frege unterscheidet entsprechend zwischen der subjektiven Bedeutung des Ganzen, den Gedanken und den Modi des subjektiven Ausdrucks dieser Inhalte insgesamt. Frege zieht eine Grenze zwischen Inhalt und Inhaltserklärung. Ein Urteil ist, wenn die Bedeutung seines Inhalts wahr ist, auch wahr und wenn sie falsch ist dann falsch. Weil das so ist, wird der Wahrheitswert von der Bedeutung bestimmt. Wie wir sehen, akzeptiert Frege die in aristotelischer Tradition stehende Korrespondenztheorie nicht, in der Wahrheit von einer äußeren Quelle des urteilenden Kontextes definiert wird. Die Frage, wie Gedanken durch Vernunft im Rahmen des Urteils wieder ergriffen werden, bleibt bei Frege unbeantwortet. Er vermeidet es über die Existenz von Gedanken zu sprechen. Der Grund dafür, dass er keine Aussagen zu diesen beiden Punkten macht, ergibt sich aus der Tatsache, dass er, wie oben erwähnt, weder die Korrespondenztheorie akzeptiert, noch der Psychologismus nicht angesprochen werden will.

\section{Die Beziehung von Sinn und Bedeutung bei Husserl}

Husserl (1859 - 1938) ist der Gründer Phänomenlogie im 20.Jahrhundert. Er hat versucht mit seiner eigenen Terminologie und seiner eigenen These offenzulegen, wie Freges Begriff vom "Gedankensinhalt" zu verstehen ist. Husserl versuchte dieses Problem zu lösen, indem er die Gefahr des Psychologismus wahrnahm und dabei von der Intentionalitätstheorie profitierte. Jedoch sind Husserls Begriff von "Inhalt" und derjenige von Frege verschieden. Was der frühe Husserl unter 'Inhalt' versteht, sind 
Sinneseindrücke, die zeitlich sequentiell, intermittierend und bewusst geschehen. Husserl nennt Sinneseindrücke Inhalt oder Vorstellung (Husserl 1992: 32). An genau diesem Punkt wurde er allerdings dafür kritisiert, objektive Entwürfe mit subjektiven Inhalten zu verwechseln. Zum Beispiel 'die Vorstellung eines Pflanzenstengels, eines Blattes oder einer Farbe (Husserl 1992b: 72)', jedes kommt von einer Teilvorstellung (Husserl 1992b:72) zueinander und bildet den Inhalt. Aus diesem Grund sind Vorstellung und Inhalt zwei empirisch fundierte Begriffe zu nennen, die bei Husserl miteinander verknüpft sind. Selbst der Psychologist Hume hat bereits "zwischen den 'impressions', den 'Sinneseindrücken', und den 'ideas', den 'Vorstellungen', unterschieden (Hume 1989:8).”

Husserl nahm an, dass es in der Außenwelt neben den Ereignissen und allen Dargestellten, die sich mir zeigen, viele verschiedene Weisen gibt und dass sie den Unterschied zwischen ihren gegebenen Bedeutungen und Wahrheiten an sich darstellen. Genau in Verbindung mit dieser Ansicht, versucht Freges Unterscheidung zwischen 'Sinn und Bedeutung', die Unterscheidung zwischen 'Sinn' und 'Vorstellung' von Dingen hervorzubringen und 'Bedeutung' durch Sinn zu erreichen.

Frege vermied es, die ontische Struktur des Gedankens zu kommentieren und darüber zu reden, ob sie direkt zu erkennen sind oder nicht. Laut Frege sind 'Gedanken' die "Begriffsinhalt", die vom Bewusstsein erfasst werden, und nicht als im Bewusstsein als konstituierende Teile gelten. Außerdem hat der konzeptuelle Inhalt kein transzendentales Verhältnis zum Gedanken. Frege unterscheidet in diesem Zusammenhang zwischen dem phänomenalen Sinn der Dinge und dem Zustand, in dem sie sich darbieten. Gleichzeitig macht er zwischen Bedeutung und Sinn eine Ableitung dieser Unterscheidung, also zwischen ihrem Wesen und ihrer Erscheinung. Frege sagt aber nichts direktes über Bedeutungen an sich aus. Während Bedeutungen nicht unmittelbar erfasst werden können, kann die Menge von ihnen sehr wohl erkannt werden. Laut Frege liefert die logische Analyse, die im Rahmen der Urteile durchgeführt wird, Wissen über die Menge jeweiliger Bedeutung, das heißt über das Verdecken von Ideen. So kann bspw. im Fall von Zahlen ihre 'Bedeutung' nicht direkt 
als ,Gedankeninhalt' eingesehen werden, sondern nur die Menge ihrer Anzahl kann über eine Menge von Begriff-Umfang (Paarungsrelation) erreicht werden. Aus diesem Grund hat Freges mathematische Philosophie nicht die phänomenologische Polarisation der Unterscheidung, Erscheinung' und ,Wesen', aber das Verhältnis von 'BedeutungSinn' ernährt die Idee der 'Intention der Bedeutung', wie sie im Kontext der Husserlschen Phänomenologie diskutiert wird. Denn nach Frege können wir, abgesehen von der Bedeutungsmenge, nur etwas über die Beziehung zwischen Sinn und Bedeutung wissen.

Husserl glaubt, dass Zahlen nur dann bestimmt werden können, wenn man den reinen Gedankeninhalt, der in Freges Definition von Zahlen enthalten ist, als Grundlage des mathematischen Wissens zurückweist. Die Rückkehr des Wissens ist nur möglich durch die reflexive Selbstorientierung, d.h. durch die Logik des Subjekts selbst. Husserl, der die Phänomenologie als Wissenschaft der Wissenschaft konstruieren will, argumentiert, dass die objektive wissenschaftliche Idee, die allen Wissenschaften zugrunde liegt, durch apophantische formale Logik realisiert werden kann. So ist die Quelle der Phänomenologie als Wissenschaft der Wissenschaften die Logik im Status der 'Semantik des Wissens' und die Bedeutung aller Wissenschaften wird durch Logik untersucht. Gemäß der Phänomenologie behandelt das Bewusstsein die Objekte als Bedeutungen.

Husserl, der die universale Bedeutung der Wissenschaft als Fundament der leibnizschen 'mathesis universalis' (gemäß der leibnizschen Vorgabe als mathesis universalis?) erforschen will, sieht in einer Reihe phänomenologischer Reduktionen eine zuverlässige wissenschaftliche Erkenntnisquelle, die objektives und apriorische Wesensallgemeinheiten in einem von psychologischen und sonstigen faktischen Elementen freien Raum bestimmt. Hier, infolge der phänomenologischen Reduktion, ist Bewusstsein kein psychologisches oder metaphysisches 'Selbst', sondern reines Bewusstsein, das an sich in der Lebenswelt mit anderen Dingen reduziert ist und somit reine Essenzen in Sinn verwandelt. Husserl achtete bei der Bewertung darauf, dass das Bewusstsein in einer Reinheit ist, die die objektive und apriorische Natur der Essenzen 
nicht überschatten kann. So führt uns die Suche nach Wissen und Wissenschaft innerhalb der Phänomenologie zur Konkretheit und damit zum Bewusstsein. Mit anderen Worten, der Versuch, die Grundlagen der Wissenschaft und ihrer Möglichkeit offenzulegen, führt Husserl zu einer Untersuchung des 'Bewusstseins', welches logische Bedeutungen schafft und für sie Sinn ergibt. Zu diesem Zweck kommt Husserl, der mit reduktiven Methoden auf das Wesentliche zurückzugreifen versucht, zu dem Schluß, dass eine Wesensschau notwendig ist, welche das Ideal direkt zum Vorschein bringt. Das heißt, nach Husserl ist reine Anschauung keine psychologische oder empirische Eigenschaft, sondern eine kategorische Anschauung, die die universale Existenzkonzeption gibt und die Möglichkeit liefert objektive Essenz zu erreichen.

Husserls objektive Grundlage, die auf arithmetischen und anderen Wissenschaften beruht, wurde durch formale analytische Überlegungen aufgeklärt. Im allgemeinen diskutierte Husserl, der die urteilende Formen zuerst mit dem Konzept der apophantischen Logik studierte, die Bedingungen der Semantik des Urteils und der Semantik im Kontext der reinen Logikgrammatik. Er behandelte danach die Urteile und Bedeutungen der 'Logik der Widerspruchslosigkeit' und untersuchte die Inferenzformen. Die formale analytische Logik bestimmt die Wahrheitswerte der Urteile, die bei der Wahrheitslogik in der Endphase ihrer Eignung des Sachverhaltens nicht entgegengesetzt sind und Widerspruchslosigkeit widerlegt werden kann. An dieser Stelle scheint Husserl die Konformitätstheorie angenommen zu haben, die Frege als Gesetz der Wahrheit und die ganze Theorie als Gesetz ablehnt.

Die Husserlsche Logik versucht insbesondere, die Bedeutungsstrukturen wissenschaftlicher Theorien zu untersuchen und Bedeutungskategorien zu bestimmen. Denn nach Husserl basieren die grundlegenden Konzepte und begrifflichen Beziehungen in den Wissenschaftstheorien auf den Bedeutungskategorien. Husserls Hauptziel ist es daher, die Bedeutungsformen zu bestimmen, die sich aufgrund von Aussagenkategorien und alten Formen $\mathrm{zu}$ neuen Bedeutungen zusammenfinden. Tatsächlich betont Husserl, der die logische Grammatik auf der Grundlage der Theorie der Bedeutungsformen studiert, in der vierten Untersuchung des zweiten Bandes der 
“Logischen Untersuchungen (Husserl 1992a)" die logische Form als Grundelement des Wissenstransfers. Es sei jedoch darauf hingewiesen, dass Husserl nicht klar zwischen der logischen Form und dem Inhalt unterschieden hat. Das zeigt sich daran, dass dasjenige, das in einem Urteil als Form gegeben ist, in einem anderen Urteil als Inhalt verwendet werden kann. Frege hingegen unterscheidet zwischen dem logischen Gehalt des objektiven Gedankeninhalts und der logischen Form. Das veranschaulicht er an Situationen, in denen sich die Einreichung nicht ändern kann, selbst wenn sich die Bedeutung des Vorschlags ändert.

Die verborgene Objektivität in den Tiefen des konzeptuellen Bewusstseins wird durch die Zurückführung auf die bewussten Grundlagen der logischen Objekte bestimmt, die mit einer kategorialen Anschauung erhalten werden. An diesem Punkt ist Husserls Annäherung an Frege im Vergleich zu ihm keine bloße Arithmetik, sondern ein Bestreben, in allen seinen Objektivitäten die Mittelbedeutungsgründe zu erreichen, durch die alle Wissenschaften zur objektiven Erkenntnis gelangen können. Husserl erforscht die Fähigkeit, Sinn in Bezug auf das reine Bewusstsein und die Sinnhaftigkeit im Rahmen der Intentionalitätstheorie zu machen; Frege hat die Objekte der Arithmetik auf dem Gebiet des objektiven Denkens positioniert und diese reinen Gedankeninhalte in einem Kontext des juristisch urteilenden Kontextes mit einer Reihe logischer Analysen der Sprache identifiziert. Husserls hauptsächlicher Unterschied zu Frege ist, dass Husserls objektive Ideale .m Gegensatz zu Freges ausdrücklicher Vermeidung, wie Ideen im urteilenden Kontext und auch in der Anschauung verfangen werden, als Mittel zum Wissen innerhalb seiner eigenen Anschauung und deren Anwendung eingesetzt werden. Er versuchte dies mithilfe der 'Intentionalität' zu erklären.

Husserl unterscheidet drei semantische Kategorien: nominale Bedeutung, adjektivierende Bedeutung und urteilende Bedeutung. Nach Husserl hängen die nominale und die adjektivierende Bedeutung von der Bedeutung der Urteilsbedeutung ab. Ihrer Beziehung aufeinander sowie ihre Positionen innerhalb des Urteils werden durch apriorische Gesetze bestimmt. Die Bedeutung der Aussage hat eine unabhängige Bedeutung und viele weitere Aussagen können aus der logischen Form des 
grundlegenden Satzes abgeleitet werden. Das Pragmatische kann zwar in das Nominale umgewandelt werden, die Nominalbedeutung und die Adjektivbedeutung können hingegen nicht synonym verwendet werden.

Husserl behandelt während seines Versuchs, die apriorischen Logikgesetze, welche Bedeutungen verbinden, zu bestimmen, auch die 'sinnlosen' Sätze, die in Unstimmigkeit syntaktischer Regeln bestehen und 'gegensätzliche Bedeutung' aufweisen (können), welche durch unsinnige Ausdrücke im Kontext von Urteilen entstehen. Husserl, der die Verwendung von Ausdrücken wie ein 'rundes Quadrat' oder ein 'geflügeltes Pferd' im Urteilskontext für bedeutungslos hält, behauptet, dass Regeln der 'Widerspruchslogik' Sinnlosigkeit verhindern würden. Die Logik der Wahrheit, die auf der Unmöglichkeit beruht, die richtigen Werte zweier widersprüchlicher Gesetze zusammenzufassen, legt Regeln nahe, die die Mehrdeutigkeit der Art und Weise verhindern, in der jede der einzelnen syntaktischen Ausdrucksformen sich befindet. Nach Husserl kann jedoch, wenn die Regeln der Wahrheit eingehalten werden, der Widerspruch vermieden werden. Der Philosoph, der versucht, die Wahrheit des Urteils eindeutig zu machen, ohne sie von der Tätigkeit des Bewusstseins, das sie enthüllt, und von dem, worauf es bezogen ist, zu abstrahieren, hält das rein scheinbare Abbild für die notwendige Zugehörigkeit. Als Bewusstseinstätigkeit findet die Logik im transzendentalen Selbst, in dem die Objektivität in sich selbst liegt, eine Quelle der Geltung und ist der allgemeinen wissenschaftlichen Idee zugänglich, die für alle Wissenschaft durch die Evidenz möglich ist. Wesen, die auf transzendentaler Ebene durch evidente Anschauung erfasst werden, werden gleichzeitig mit den apriori Bedeutungen im Bewusstsein bemerkt. Evidenz ist der Akt, der dies ermöglicht. Evidenz ist kein mytisches Erlebnis, es intendiert die Wesen von Ideationsobjekten, welche beständig reduziert werden. Diese rationale Anschauung leitet sich aus dem Bewusstsein und der Abstrahierung des Vernünftigen ab. Obwohl Husserl behauptet, dass anschaulicher Inhalt, der ein Noema erfüllt, reine Anschauung sei, die frei ist von allen psychischen und sinnlichen Elementen, gibt es keinen Konsens darüber, dass Husserls Verständnis der Idee nicht von den empirischen Grundlagen entfernt ist. 
Die 'Begriff-Objekt'-Relation, die bei Frege auf der 'Funktion-Argument'Unterscheidung basiert, wird von Husserl als 'Intentionalität' auf der Basis der 'NoemaNoesis' Unterscheidung aufgegriffen. Husserl folgt eher der Vorstellungserkenntnis von Mill und Bolzano, statt derjenigen Kants und von der Hinsicht, dass sie auf Intentionalität basiert folgt Husserl Frege. Die beiden Konzepte, die sich in der Regel ähneln, sind durch den Husserls 'Noema' Begriff und Freges Bedeutung voneinander getrennt. Bei Frege bedeutet 'Bedeutung', das Objekt in einem bestimmten Kontext auszudrücken; bei Husserl hingegen ist Noema die Verallgemeinerung des Begriffs der Bedeutung für den Bereich aller Handlungen. Wir können es auch klarer formulieren: Wenn ich etwas wahrnehme ist meine Wahrnehmung eine von vielen; genau das was ich unter vielen Möglichkeiten wahrnehme ist laut Frege der ,Sinn'. Die Menge von allen Möglichkeiten der Wahrnehmungen des Dinges hingegen ist laut Husserl etwas anderes als der 'Noema'.

Für Husserl bezieht sich Freges Definition von 'Sinn' und seine Definition im Kontext der Sprache, auf eine nichtsprachliche Quelle: reine Anschauung. Bei Frege hingegen gibt uns 'Sinn' eine objektive und logische Bedeutung. Husserl benutzt Freges 'Bedeutung' anstelle von 'Sinn'. Nach Husserl bedeutet 'Noema' als 'Bedeutung' die sprachlichen Handlungen und Ausdrücke; es besteht aus dem Gegenstand und wandelt Daten über das Objekt in Anschauungen um. Das Wort 'Sinn' wird von Husserl als 'breiterer Sinnbegriff für alle Handlungen' gebraucht. 'Bedeutung' ist bei Husserl, anders als bei Frege, nur im Kontext von 'Ausdrücken' nutzbar. Husserl verwendet den Begriff 'Gegenstand' anstelle von Freges 'Bedeutung'. Er vermeidet, ähnlich wie Frege, über dieses Thema zu kommentieren. Das einzige, was nach Husserl getan werden kann ist, durch Reflexion zu der vorgefundenen Lebenswelt zurückzukehren und die Erscheinungen von Phänomenen 'vor-Noema' auf dem Gebiet der Wesen zu bestimmen. Freges 'Erfassung objektiver Denkinhalte' bezieht sich somit auf den Erwerb reiner Ansprüche bei Husserl im Bereich der transzendentalen Wesen.

Einer der Punkte, an dem sie sich ähneln ist, dass sowohl Husserls Noema als auch Freges Sinn objektive und ideale Strukturen sind. Eine weitere Ähnlichkeit 
zwischen den beiden Philosophen besteht darin, dass Husserl das 'Noema' vom Gegenstand getrennt hat, ähnlich wie Freges 'Sinn-Bedeutung' Unterscheidung. Hier sind allerdings nicht Husserls 'Gegenstand' und 'der intendierte noematische Gegenstand' das Wichtige, sondern dass der Sinn, von dem das Noema stammt, auf den Gegenstand deutet. Sowie bei Frege 'Bedeutung' ein von ,Sinn' Verschiedenes ist, so ist bei Husserl 'Gegenstand' ein von 'Bedeutung' Verschiedenes. Beide sehen also einen Unterschied zwischen Sinn und Bedeutung.

'Noema' ist als objektive Bedeutung in der Husserl-Terminologie ist kein Objekt, das real im Bewusstsein enthalten ist, obwohl es inhärent von bewussten Handlungen abhängig ist, sondern eher Gegenständlichkeit. Weil Intentionalität die Relation zwischen Bewusstsein und dieser Gegenständlichkeit bewirkt, werden während einer noetischen Aktanalyse (aktmäßige Analyse) neben reinen Erlebnissen auch noematische Strukturen behandelt. Aus diesem Grund wird die eidetische Analyse sowohl in 'Noesis' als auch 'Noema' Feldern entsprechend der bilateralen Struktur der Intentionalität durchgeführt. Daher hat sich das reflexive Urteil und die Logik der Phänomenologie zum 'Sinn' gewandelt. Noema trägt auch als die Gegenständlichkeit von Intentionalität seine eigene Objektivität und ihren eigenen Sinn mit sich. Der Sinn von Noema ist der Sinn von Bedeutung und wird in einer vorübergehenden Ebene aufgedeckt, in der das Bewusstsein reflexiv auf seine bewussten Handlungen ausgerichtet ist. So werden die Vorstellung und das Bewusstsein der Welt durch die Wirkung des apriorischen Wesen der ihnen entsprechenden Bedeutungen und Ausdrücken erreicht.

Husserl konzentriert sich auf Inhalte, die sich auf das Wesen der Rede beziehen, und hinterlässt die logische Form, die in den 'Ideen' die Form des Erzählens vermittelt, indem er es durch Noema-Noesis erklärt. Gemäß dieser Unterscheidung gibt es eine Unterwerfung von Aussagen, in denen es möglich ist, dass die Erzählung als Apriori auftritt. Bei Vorstellungen wird Einbildungskraft, die nicht apriorisch realisiert werden kann, als 'sinnlos' betrachtet. Trotzdem können Ausdrücke ohne Sinn dank dem Noema bedeutungsvoll sein. Die Gegenstände der Arithmetik sind nach Husserl leere Noemata, die im Verlauf der Zeit mit kategorischen / kategorialen Anschauungen gefüllt werden. 
Ähnlich wie bei der Annahme, dass laut Frege eine Funktion mit einem Argument vervollständigt wird, schlägt Husserl vor, dass das leere 'Noema' einer 'Noese' entsprechen würde, nachdem es mit einer Anschauung erfüllt wurde. Statt der Funktion, die Freges Träger der Bedeutung ist, ist also das leere Noema bei Husserl der Träger der Bedeutung. Nach Husserl beruht die Wahrheit arithmetischer Sätze niemals auf empirischer Adäquatheit. Die in arithmetischen Sätzen ausgedrückten Idealitäten beruhen auf den Gesetzen der logischen Wahrheit, die unabhängig vom Bewußtsein sind. Mit einer “objektiven Theorie der Erkenntnis [...], einer reinen Phänomenologie der Denk- und Erkenntniserlebnisse (Husserl 1992a: 6)" trug Husserl den Bereich der Objekte außerhalb der psychologischen und empirischen Recherchebereiche. Husserl verteidigt folgendes: Die "reine Phänomenologie der Erlebnisse überhaupt [...] hat es ausschließlich mit den in der Intuition erfaßbaren und analysierbaren Erlebnissen in reiner Wesensallgemeinheit zu tun, nicht aber mit empirisch apperzipierten Erlebnissen als realen Fakten, als Erlebnissen erlebender Menschen oder Tiere in der erscheinenden und als Erfahrungsfaktum gesetzten Welt (Husserl 1992a: 6)." Trotzdem bleibt er bei Analysen der Erlebnisse empirischen Phänomenen treu. Elisabeth Ströker klärt dies folgendermaßen auf:

\footnotetext{
"Zwar mußte in einer solchen Wesensanalyse allemal auf konkrete Erlebnisse eines individuellen Bewußtseins rekurriert werden; doch geschah dies nicht, wie in der Psychologie, mit der Zielsetzung, zu bestimmten Klassifikationen oder einer bestimmten Typik von realen Bewußtseinseigentümlichkeiten zu kommen. Vielmehr fungierten darin die Einzelerlebnisse realer Personen nurmehr exemplarisch zu dem Zweck, daß auf solchem 'exemplarischen Grund' mögliche Wesenseinsichten über apriorische Bewußtseinsstrukturen erlangt werden konnten (Ströker 1987:97).”
}

Die Husserlsche Phänomenologie behandelt die Objekte der Arithmetik durch die 'Noema-Noesis' Unterscheidung. Mit anderen Worten, arithmetische Objekte gewinnen Form, wenn sie sich durch das 'leere x', was ein Bedeutungsverleihendes Merkmal des Bewusstseins ist, einem Objekt nähern. Das 'leere x', das ein logisches Phänomen ist, ist die Quelle der Klarheit. 'Leeres x' ist die logische Möglichkeit von Informationen. So führt der Rückzug aus der Welt durch Reduktionen der Konzeption der Lebenswelt zu leeren logischen Formen, in denen phänomenologische Inhalte geleert werden. Auf diese Weise wird ein Übergang von einer sinnlichen Raum-Zeit Ebene zu einer 
formalen Apriori Ebene gemacht. Apriori-Idealitäten, die das Forschungssubjekt der Logik und Arithmetik sind, können auf das reale Feld angewendet werden, auch wenn sie unabhängig von der Lebenswelt sind. Zum Beispiel kann, obwohl alle Zahlen ideal sind, die mit ihnen durchgeführte Zählung auf das reale Feld angewandt werden.

\section{Schluss}

Infolgedessen hat das Problem der Bedeutung, das im Prozess der Erlangung der objektiven Arithmetikbasis über die formale Sprache entsteht, zur Ausbeutung der Arithmetik geführt, die im Sinne phänomenologischer Erklärungen versucht wird, logisch begründet zu werden. Die Wirkung von Frege auf Husserl ist die zwischen Sinn und Vorstellung zu unterscheiden und die Objektivität der Bedeutung $\mathrm{zu}$ retten: Husserls Noema ist eine generalisierte Version von Freges Sinn. Er griff Freges Überlegungen zu ,sinnlosen Objekten“ auf und setzte sie in seiner Terminologie um als das Bedeutung verleihende Noema und den Gegenstand der Bedeutung.

Die Partnerschaft zwischen Frege und Husserl auf der Grundlage von Objektivität und die Erlangung einer fundierten Darstellung bestimmter Kenntnisse hat aufgrund ihrer unterschiedlichen Qualitäten zur Etablierung unterschiedlicher Philosophien geführt. Frege hat die Grundlagen der Objektivität und damit die arithmetischen Objekte, in einer objektiven Struktur außerhalb der Raum-Zeit, sowie außerhalb des mentalen und nichtraumzeitlichen Faktisches verortet. Husserl hingegen lehnte Freges Kritik am platonischen Realismus ab und versuchte im Bereich der reinen transzendentalen Essenz die Grundlagen der Objektivität zu finden. Um Mehrdeutigkeit bei Bedeutungen $\mathrm{zu}$ beseitigen und das Objekt $\mathrm{zu}$ bewahren, hat Frege eine formale Logiksprache als Werkzeug benutzt, während Husserl alle relevanten Urteile über Existenz und empirische Vernunft in einer Reihe phänomenologischer und eidetischer Reduktionen eingeklammert hat. Beide Philosophen profitieren letztlich von reiner Logik, bei ihrem Versuch sicheres Wissen zu begründen. Sie versuchten ihre Forschung auf einem objektiven Grund zu realisieren. Der Punkt, der Husserl und Frege voneinander unterscheidet, ist, dass Husserl dies versucht, indem er sich der Tiefe des 
Subjekts zuwendet und die reine Objektivität, die dort liegt, findet und so das Subjekt stärkt, während er versucht, die Objektivität zu sichern. ${ }^{2}$ Frege hat versucht, auf dem objektiven Boden zu bleiben, indem er das Thema völlig außer Frage stellt. Frege hat einen wesentlichen Beitrag zur Geschichte der Philosophie des 20. Jahrhunderts geleistet, indem er konzeptionelle Erklärungen für die Gegenstände und Prinzipien der Arithmetik gegeben hat, gleichwohl er damit metaphysische Schwierigkeiten verursachte, weil wir die Objektivität vermieden hat um nicht in die Falle vom Psychologismus zu geraten. Frege argumentiert ferner, dass die Arithmetik aus der Logik abgeleitet werden könne und behandelt sie auf einer apriorisch analytischen Basis. Husserl hingegen argumentiert, dass Logik und Arithmetik die Formalitäten sind, die entstehen, wenn das Bewusstsein sich selbst zuwendet und seine eigenen Phänomene erforscht. Genauer gesagt, Husserl hat keine vernünftige Sichtweise auf die Objektivität von Arithmetik und Logik, mit einem arithmetischen Verständnis, das noch nicht aus seinen empirischen und subjektiven Grundlagen herausgerissen wurde entwickelt.

Die beiden philosophischen Traditionen wurden in dieser Arbeit mit verwandten Punkten behandelt. Die Aspekte der phänomenologischen Interpretationen der Arithmetik, die sich auf die Sinnfrage beziehen, wurden in den Vordergrund gezogen. So sind die phänomenologischen Betrachtungen auf den Grundlagen des Sinnproblems, das wir mit Frege und Husserl allgemein zu erklären versuchten, im Rahmen der Beziehung von Mathematik, Logik und Sprache behandelt worden. Wie wir gesehen haben, haben Frege und Husserl versucht, in jeweils eigener Terminologie, zwischen Sinn, Bedeutung, Vorstellung und Gegenstand zu differenzieren, um so den Begriff der Bedeutung zu verstehen und seine Objektivität zu sichern. In diesen Bemühungen rettete Frege die Objektivität der Bedeutung, indem er nicht nur zum Zeitpunkt der

${ }^{2}$ Objektivität ist nicht allein vom vereinzelten Subjekt verbürgt, sie ist eine Sache der Intersubjektivität, d.h. mehrerer Subjekte als interagierende. Eine objektive Gegenständlichkeit (etwa Geometrie) kann evident gestiftet sein (als originales Erlebnis) und persistiert ferner, weil es sich immer wieder evident (als reproduziertes originales Erlebnis) reaktiveren lässt. Kommuniziert und nacherlebt von Subjekten qua Mitteilungskonnex bewährt sie sich als objektiv. Schriftlich fixiert kann sie über die jeweilige Lebensdauer des urstiftenden Subjekts und seiner Gesprächspartner hinaus eine Überzeitlichkeit konstituieren $\rightarrow$ Objektivität (jeweiliger Sache). 
Bedeutsamkeit im logischen Raum blieb. Husserl wurde vorgeworfen, er habe die objektiven Grundlagen der Philosophie der kategorischen Sicht nicht zeigen können. Er habe psychische Akte auf psychologische Vorgänge reduziert und sei deswegen in die Falle des Psychologismus getreten. So hat er bspw. vertreten, dass mathematische Begriffe und Operationen, wie Zählen und Summieren, nur durch psychische Akte möglich sind: laut Husserl ist "Kolligieren (Husserl 1992b: 146)" als Einigung von konkreten Inhalten zu Inbegriffen (Husserl 1992b:146) eine psychische Tätigkeit. (Also stimmst du nun zu, dass Husserl in die ,psychologistische Falle' getappt ist?) Es ist offensichtlich, dass das Problem der Bedeutung für Husserl wie für Frege ein sehr wichtiges ist, das die beide Philosophen auf vielerlei Weisen untersuchen; von Logik und Wahrheit über Objektontologien bis hin zu Beziehungen mit Psychologismus und Methoden. Wir versuchten in dieser Arbeit zusammenzufassen, wie und in welchem Zusammenhang die beiden Philosophen den Begriff der Bedeutung behandeln und worin er sich unterscheidet. 


\section{(Genişletilmiş) Türkçe Özet}

Frege'nin başını çektiği analitik felsefe geleneğinde aritmetiğin nesnel temellerinin formel dil üzerinden elde edilmesi sürecinde ortaya çıkan 'anlam sorunu', mantıksal olarak temellendirilmeye çalışılan aritmetiğin, fenomenoloji açısından verimli açıklamalarla ele alınmasına yol açmış ve Frege tarafından Husserl'e yöneltilen eleştirilerle fenomenolojinin olgunlaştırılmasına katkı sunmuştur. Husserl, anlamın nesneyle bağıntısını açık kılmak adına Fregeci anlam görüşünün geliştirilmiş versiyonu olan, tüm anlamları kuşatıcı mânâ olarak 'noema' düşüncesini ortaya atmıştır. Doğruluk değerinden yoksun ifadeleri Frege'nin yaptığı gibi gönderimsiz olmakla değil, 'noesis'ten yoksun olmakla kategorize eden Husserl, ifadelerin anlamlı olma ölçütünü, 'noema', doğruluk değeri alma koşulunu ise 'noesis' olarak tayin etmiştir. Dolayısıyla noema sayesinde anlamı, bir nesneye sahip olup olmama durumundan bağımsız biçimde ele alan filozof, bilinç Intention'larının görüsel bir yaşantıyla tamamlanması hâlinde doğru ya da yanlış olabildiklerini, aksi hâlde anlamlı olmalarına karşın doğruluk değerinden yoksun olacaklarını kabul etmektedir. Aynı şekilde Frege de gönderimi (Bedeutung) olan her ifadenin doğruluk değeriyle birlikte aynı zamanda bir anlam (Sinn) sahibi olmasına karşın, anlamlı olan her yargının bir doğruluk değeri almak zorunda olmadığını savunmakla Husserl'e yakın bir görüş benimsemektedir. Farklı Husserl ile Frege arasındaki en büyük fark, Husserl'in doğruluk değeri alma ölçütü olarak gördüğü görüsel yaşantıya, Frege'nin başvurmamış olmasıdır. Öte yandan kullandıkları terminoloji bakımından da ayrılan Husserl ile Frege'de anlam, doğruluk ve nesne, farklı gelenek ve üslûplar üzerinden tartışılmıştır. Öyle ki Frege'deki gönderime (Bedeutung); Husserl'deki nesnenin (Gegenstand); Frege'deki anlama (Sinn) ise Husserl'deki Bedeutung'un karş1lık düştügü görülmektedir. Frege'den farklı olarak Husserl'in Sinn kavramına biçtiği rol ise Türkçe'de en iyi „mânâ“" kelimesiyle karşılanabilmektedir. Zira Husserl'deki Sinn, tümel anlam olarak düşünülmektedir. Böylece adlararasılıktan anlamlararasılığa geçişte anlam ile nesne, ad ile anlam ve ad ile nesne arasındaki bağıntılar bizi semantik bir alana taşımaktadır. Zira dilde adını koyduğumuz her şeyin, sahip olduğu anlamı, içerik üzerinden mi, tasarım üzerinden mi, gönderim üzerinden mi yoksa dilin kullanım bağlamı üzerinden mi kazandığı sorusu, nesnel bilginin olanağını ve dil aracılığıyla ifade edilebilme koşullarını ortaya koyabilmek açısından önemli bir sorudur. Fakat bu soruyla bağlantılı olarak dilin nesnel anlamı nasıl temsil edebildiği sorusunu da yanıtlamak gerekir ki bu soruyu yanıtlayabilmek için dil ile bilinç arasındaki ilişkinin analiz edilmesi 
gerekmektedir. Husserl bu analizi olgu bireşimleri üzerinden, Frege ise düşünceler üzerinden kurmaktadır. Husserl fenomenolojisinde Intention üzerinden, Frege'nin başını çektiği analitik perspektifte ise kavram-kaplam ilişkisi üzerinden temellendirilen bu bağ, bilinçteki nesnel anlamların dilde adlara karşılık gelecek şekilde kullanılabilme ve bir anlam sahibi olabilme olană̆1 olarak görülmektedir.

Frege nesnelliğin ve dolayısıyla da nesnel yapıdaki aritmetik nesnelerin temellerini, zihinsel ve olgusal olanın dışındaki uzam-zaman dışı bir düşünceler aleminde konumlandırırken Husserl ise nesnelliğin temellerini saf eidetik alandaki transendental anlamlar üzerine inşa etmektedir. $\mathrm{Bu}$ yolda karşılaşlabilecek anlam belirsizliklerinin ortadan kaldırılması ve nesnelliğin muhafaza edilmesi için Frege formel bir mantık dilini araç edinmiş, Husserl ise bir dizi fenomenolojik ve eidetik indirgeme ile varoluş ve empirik nedensellikle ilgili tüm önkabullerle birlikte dili de paranteze almış, düşünceye konu olan her şeyi içeriksizleştirip saflaştırmıştır. Neticede her iki filozof da nesnel bilgiyi temellendirmeye çalışırken saf formel bir dil ile iş görmüş, mantık kılavuzluğunda ilerlemiş ve araştırmalarını dolaysız bir kesinlikle gerçekleştirmeye çalışmıştır. Husserl ile Frege'nin ayrıldıkları temel nokta ise Husserl'in bunu yapmaya çalışırken öznelliğin derinliklerine dönmek suretiyle orada yatan saf nesnelliği bulup çıkarması, dolayısıyla da nesnelliği güvenceye almaya çalışırken öznelliği güçlendirmesi; Frege'nin ise öznelliği tamamen konu-dışı ilan ederek nesnel zeminde kalmayı başarmış olmasıdır. Öte yandan Husserl kimi eserlerinde yer yer anlam ile tasarım arasındaki ayrımı koruyamadığı için eleştirilmekte, Frege ise aynen öznellikte olduğu gibi tasarım konusunda da bunların aritmetik, mantık ve felsefe alanından uzaklaştırılması gerektiğine inandığı için tasarımları peşinen konu dışı ilan etmektedir. Her ne kadar Frege, psikolojizme düşmemek pahasına, düşünceler alanında bulunan mantıksal nesnelerin bizim tarafimızdan nasıl yakalandığını açıklamaktan kaçındığı için metafizik sıkıntılara sebebiyet vermişse de, aritmetiğin nesne ve ilkelerinin kavramsal açıklamasını sunmak bakımından 20.yy felsefe tarihinde başat bir konumda görülmektedir. Ayrıca Frege, aritmetiğin mantıktan türetilebileceğini savunup onu apriori analitik zeminde ele alırken Husserl ise mantık ve aritmetiğin, bilinçteki saf eidetik özlerle iş gören formel bilimler olduğunu savunmuştur. Çalışmamızın genelinde adlararasılıktan anlamlararasılığa evrilen güzergâhlarıyla bu iki felsefî gelenek, aritmetiğin, mantık ve dil ile olan ilişkisi üzerinden ele alınmıştır. Frege, nesnel anlamı mantıksal zeminde elde etmeye çalışırken Husserl anlamın dolaysız bir apaçıklıkla kavranılmasını temellendirmeye çalışırken başvurduğu 'kategorik görü’ savıyla nesnel bilgiyi 
Diler Ezgi TARHAN, “Von der Analytischen Philosophie zu der Phänomenologie: Von Internamen zu den Intersinnen ," Kaygl, 19(1)/2020: 152-180.

hâlâ empirik bir yaşantı üzerinden elde ettiği gerekçesiyle eleştirilmiştir. Böylece bu makaleyle analitik adlararasılıktan fenomenolojik anlamlararasılığa geçişte saf transzendental anlamların reflektif Intention'u üzerinden anlamın dilde açığa çıkma koşulları betimlenmeye çalışılmıştır. 


\section{BIBLIOGRAPHIE}

\section{Leipzig.}

BOLZANO, Bernard (1929). "Wissenschaftslehre", Felix Meiner Verlag,

FREGE, Gottlob (1884). "Die Grundlagen der Arithmetik. Eine logisch mathematische Untersuchung über den Begriff der Zahl", Verlag von Wilhelm Koebner, Breslau.

FREGE, Gottlob (1918-1919). "Der Gedanke. Eine logische Untersuchung. Beiträge zur Philosophie des deutschen Idealismus - II”, ss.58-77. 21.12.19, (http://www.gavagai.de/texte/HHPTX01.htm).

FREGE, Gottlob (1964). "Begriffsschrift. Über den Zweck der Begriffsschrift", Hildseim, Geroge Olms.

FREGE, Gottlob (1994a). "Funktion und Begriff": Funktion, Begriff, Bedeutung. Fünf logische Studien”, Hrsg. und eingel. Von Günther Patzig. 7.Nachdruck, Vandenhoeck und Ruprecht, Göttingen, ss.18-39.

FREGE, Gottlob (1994b): "Über Sinn und Bedeutung: Funktion, Begriff, Bedeutung: Fünf logische Studien”. Hrg: Günther Patzig. 7. Auflage, Vandenhoeck und Ruprecht, Göttingen, ss.40-65.

FREGE, Gottlob (1998). "Begriffsschrift und andere Aufsätze. Zweite Auflage. Mit E. Husserls und H. Scholz' Anmerkungen", herausgegeben von Ignacio Angelelli. 5. Nachdruck der 2. Auflage. Georg Olms Verlag, Hildesheim.

HUME, David (1989). "Ein Traktat über die menschliche Natur, Erstes Buch: Über den Verstand", mit neuer Einführung herausgegeben von Reinhard Brandt, Hamburg.

HUSSERL, Edmund (1992a). "Logische Untersuchungen I", Gesammelte Schriften, Band IV, Elisabeth Ströker, Meiner, Hamburg.

HUSSERL, Edmund (1992b). "Philosophie der Arithmetik. Logische und psychologische Untersuchungen, Erster Band", Gesammelte Schriften, Band-I, herausgegeben von Elisabeth Ströker, Meiner, Hamburg.

STRÖKER, Elisabeth (1987). “Phänomenologische Studien”, Frankfurt am Main.

WHITESITT, J.E. (1973). "Boolesche Algebra und ihre Anwendungen - Logik und Grundlagen der Mathematik", Vieweg Verlag, Braunschweig.

WITTGENSTEIN, Ludwig (1984). "Philosophische Untersuchungen”. In: "Ludwig Wittgenstein. Werkausgabe Bd. I', Suhrkamp, Frankfurt am Main, ss.225-589. 